Journal Club

Editor's Note: These short, critical reviews of recent papers in the Journal, written exclusively by graduate students or postdoctoral fellows, are intended to summarize the important findings of the paper and provide additional insight and commentary. For more information on the format and purpose of the Journal Club, please see http://www.jneurosci.org/misc/ifa_features.shtml.

\title{
NMDA Receptor Excitotoxicity: Impact on Phosphatase Activity and Phosphorylation of Huntingtin
}

\author{
Michael R. Jablonski, ${ }^{1,2}$ Lori Cooper, ${ }^{1}$ and Dena A. Jacob ${ }^{1,2}$ \\ ${ }^{1}$ Department of Neuroscience and ${ }^{2}$ Weinberg Unit for ALS Research, Thomas Jefferson University, Philadelphia, Pennsylvania 19107 \\ Review of Metzler et al.
}

Huntington disease (HD) is a late-onset neurodegenerative disorder characterized by the loss of striatal and cortical neurons, motor abnormalities, emotional disturbance, and cognitive dysfunction. The disease results from an expansion of a CAG sequence in the huntingtin gene beyond the normal allelic range of 10-35 CAG triplets. This CAG triplet repeat encodes a polymorphic polyglutamine tract near the $\mathrm{N}$ terminus of the huntingtin (htt) protein. Insoluble inclusions of polyglutamine (polyQ)-expanded htt (mhtt) are common hallmarks of $\mathrm{HD}$, although the inclusions are likely not toxic to the cell (Arrasate et al., 2004). The toxic species (e.g., dimers or oligomers) of mhtt and the pathogenic mechanism of polyQ-expanded htt underlying HD are unknown.

Excitotoxicity mediated by NMDA receptors (NMDARs) has been suggested to play a role in HD-associated neuron death (Okamoto et al., 2009). In particular, evidence suggests that activation of extrasynaptic NMDARs is necessary for inclusion formation and cellular toxicity (Okamoto et al., 2009). In a mouse model of HD, selectively blocking extrasynaptic NMDARs with low doses of the NMDA inhibitor memantine promoted aggregation and

Received Dec. 26, 2010; revised Jan. 20, 2011; accepted Jan. 20, 2011. We thank Dr. Manuel Covarrubias, Dr. Diane Merry, and Dr. Davide Trotti for reviewing and editing this commentary.

Correspondence should be addressed to Michael R. Jablonski, Jefferson Hospital for Neuroscience, Weinberg Unit for ALS Research, 900 Walnut Street, 451, Philadelphia, PA 19107. E-mail: michael.jablonski@jefferson.edu. DOI:10.1523/JNEUROSCI.6747-10.2011

Copyright $\odot 2011$ the authors $\quad 0270-6474 / 11 / 314357-03 \$ 15.00 / 0$ decreased toxicity of mhtt (Okamoto et al., 2009). These effects are thought to be achieved through restoration of PGC1- $\alpha$ function induced by increased CREB activity.

Although excitotoxicity appears to be a component of HD pathogenesis, recent papers have highlighted the importance of htt phosphorylation in reducing toxicity of polyQ-expanded htt. Phosphorylation of S13 and S16 decreases aggregation and toxicity of mhtt in both cell and mouse models of HD (Gu et al., 2009), perhaps via disruption of an amphipathic helix at the $\mathrm{N}$ terminus and subsequent interference with self-association of the hydrophobic protein face. In wild-type (WT) mice, phosphorylation of the $\mathrm{S} 421$ residue (pS421-htt) is decreased in the striatum, the region most susceptible in $\mathrm{HD}$, relative to both the cerebellum and the cortex. Furthermore, human frontal cortex displays endogenous phosphorylation of S421-htt under physiological conditions (Warby et al., 2005). Akt phosphorylation of this residue prevents toxicity induced by mhtt (Humbert et al., 2002) and restores anterograde and retrograde axonal transport in neurons (Zala et al., 2008). Phosphorylation of S421 also prevents cleavage of mhtt by caspase 6 (Warby et al., 2009), a step that is thought to be necessary for toxicity (Graham et al., 2006).

A paper by Metzler et al. (2010) connects NMDAR excitotoxicity with pS421htt in HD progression. Primary cortical neurons from YAC128 transgenic mice, which express mhtt, were more susceptible to NMDAR-mediated dephosphorylation of S421-htt than WT neurons. Moreover, the authors observed the selective decrease of pS421-htt in the striatum of YAC128 mice throughout disease progression; no decrease in pS421-htt was detected in relatively unaffected areas such as the cerebellum. Control animals did not show a decrease of pS421-htt in the striatum or cerebellum throughout disease.

To address underlying mechanisms mediating decreased pS421-htt, the authors examined potential changes in Akt kinase activity. Akt was previously shown to phosphorylate S421-htt (Humbert et al., 2002; Warby et al., 2005), so a decrease in pS421-htt could result from decreased Akt activity. However, upon NMDA treatment, there were no significant differences in Akt kinase activity between WT and YAC128 primary cortical neurons. Interestingly, Akt activity significantly increased in both genotypes after treatment with low doses of NMDA. Metzler et al. (2010) speculate that selective activation of NMDAR may increase Akt activity and neuronal survival. For example, since synaptic and extrasynaptic NMDARs preferentially affect neuronal protection and cell death, respectively (Hardingham and Bading, 2010), the significant increase in pS473-Akt expression levels detected after low-dose NMDA treatment could result from activation of synaptic NMDARs. In contrast, the return to baseline of pS473-Akt expression levels upon treatment with higher NMDA concentrations could involve both synaptic 
and extrasynaptic NMDARs. This hypothesis was tested by Milnerwood et al. (2010) using chronic low-dose memantine treatment. CREB activity, a downstream target of Akt, increased in the striatum of YAC128 mice with low-dose memantine treatment compared to WT and untreated YAC128 mice.

Metzler et al. (2010) corroborated these data in vivo by evaluating pS421-htt in WT, YAC128, YAC18 (nonpathological htt), and C6R (caspase-6-resistant) mice after intrastriatal injections of the NMDAR agonist quinolinic acid (QA). After 15 min of QA-induced excitotoxicity, pS421-htt levels were significantly reduced in striatal lysates from YAC128 compared to WT, YAC18, and C6R mice [Metzler et al. (2010), their Fig. 2]. Moreover, both YAC18 and C6R mice, which are relatively resistant to QA-induced excitotoxicity (Graham et al., 2006) showed an early increase in pS421-htt. These experiments demonstrate a relationship between excitotoxicity and phosphorylation of S421-htt.

Because the activities of the kinases responsible for S421-htt phosphorylation were unchanged, the authors analyzed phosphatase activity. In transiently transfected COS7 cells and WT cortical neurons, the phosphatases PP1 and PP2A potently dephosphorylated S421-htt, whereas PP2B (calcineurin) did not significantly contribute to reducing pS421-htt [Metzler et al. (2010), their Fig. 4B]. However, previous evidence from Pardo et al. (2006) using primary striatal neurons transfected with an $\mathrm{N}$-terminal fragment of htt (480-17Q) and a constitutively active form of calcineurin demonstrated a reduction in pS421-htt. Pardo et al. (2006) also confirmed this finding in vivo, where administration of $\mathrm{PP} 2 \mathrm{~B}$ inhibitor increased pS421-htt in WT whole-brain homogenate (Pardo et al., 2006). Calcineurin activation depends on the presence of $\mathrm{Ca}^{2+}$, which may account for the conflicting findings and, given this discrepancy, Metzler et al. (2010) emphasize the importance of PP1 and PP2A in S421htt dephosphorylation in vitro without excluding contribution from PP2B. Analysis of PP1 and PP2A inhibition in vivo will be necessary to fully determine their role in pS421-htt modulation after excitotoxic stimulation. Furthermore, although PP1, PP2A, and PP2B are the most common phosphatases in the brain, use of the nonspecific PP1 and PP2A inhibitor okadaic acid (OA) (Mansuy and Shenolikar, 2006) in the current experiments does not exclude the involvement of other phos- phatases. Finally, additional studies are necessary to confirm the primary effects of okadaic acid on S421 phosphorylation. For example, if pS421 is directly regulated by $\mathrm{OA}$, nontransgenic striatal neurons expressing the S421 phosphonull (S421A) or phosphomimetic (S421D) constructs (Warby et al., 2009) treated with NMDA and glycine would be resistant to OA.

Given the ability of PP1 and PP2A to dephosphorylate S421-htt, the authors extended their analysis by examining whether phosphatase inhibition protected neurons. Cell death was significantly increased in YAC128 primary cortical neurons compared to WT after NMDA-induced excitotoxicity. Cotreatment with OA inhibited the loss of pS421-htt, resulting in decreased cell death in both neuronal types [Metzler et al. (2010), their Fig. 5]. Despite the somewhat indirect methods of cell death analyses (i.e., caspase 3 activation and evaluation based on morphological criteria), the finding that increased pS421-htt levels positively impacts neuronal protection is, nonetheless, exciting.

Finally, because in situ results suggested that $\mathrm{PP} 1$ and $\mathrm{PP} 2 \mathrm{~A}$ regulate the phosphorylation status of S421, the authors investigated whether changes in pS421-htt levels resulted from altered regulation of phosphatase activity. Decreased expression of Darpp-32, a potent inhibitor of PP1 that integrates dopaminergic and glutamatergic inputs, occurs throughout disease progression in the striatum of other HD mouse models (Van Raamsdonk et al., 2006). Decreased expression also occurred in the striatum of YAC128 mice but not in YAC18 and C6R mice [Metzler et al. (2010), their Fig. 7]. Metzler et al. (2010) found that $D_{1} R$ stimulation, which activates Darpp-32, increased pS421-htt in YAC128 mice, suggesting that dopamine signaling could help regulate S421 phosphorylation through Darpp-32. Based on these observations, Darpp-32 could be a possible link between the decrease in pS421-htt, dopaminergic and glutamatergic signaling, and phosphatase activity; however, additional studies are necessary to further understand its role in these processes.

The wider impact of this paper is illustrated by the recent report of two additional findings from the same group that emphasize the importance of excitotoxicity in HD. Milnerwood et al. (2010) reported that the prolonged treatment of YAC128 mice with a low dose of the extrasynaptic NMDAR antagonist memantine raised striatal pS133CREB levels. Given that CREB activation is critical to neuronal survival and in light of the observation by Metzler et al. (2010) that
pS133-CREB decreases in striatal homogenates from YAC128 mice throughout disease progression (their Fig. 8), it is possible that phosphatase activity is differentially regulated by synaptic and extrasynaptic NMDAR pathways, the latter of which leads to toxicity of mhtt in HD. Thus, an evaluation of differences in phosphatase activity relative to NMDAR localization and excitotoxicity may reveal interesting therapeutic strategies. Second, Graham et al. (2010) showed that NMDA stimulation significantly increases caspase-6 activity in YAC128 striatal neurons compared to WT neurons. Together with the findings of Metzler et al. (2010), not only does pS421-htt protect against NMDA-induced excitotoxicity, but caspase- 6 activity is triggered by NMDA-mediated excitotoxicity, which leads to cell death. Interestingly, activation of the caspase cascade can be suppressed by activating synaptic NMDARs, and increased activity of extrasynaptic NMDARs leads to neuronal dysfunction and death (Hardingham and Bading, 2010). Future experiments to confirm this connection and explore this cellular balance may reveal new ways to therapeutically target $\mathrm{HD}$.

In conclusion, Metzler et al. (2010) have provided evidence that NMDAR-mediated excitotoxicity regulates pS421-htt in HD, furthering our understanding of pathogenic mechanisms underlying this disease. NMDAR stimulation significantly reduced pS421-htt in isolated YAC128 neurons, and pS421-htt was also decreased after QA treatment in vivo. This suggests an enhanced susceptibility to NMDAR-mediated excitotoxicity that may contribute to the progressive loss of pS421-htt in these mice. After ruling out variations in kinase activity, the authors examined regulation of phosphatases to determine the cause of decreased pS421-htt. While the phosphatases PP1 and PP2A dephosphorylated S421-htt in vitro, additional experiments are necessary to determine both the role of $\mathrm{PP} 2 \mathrm{~B}$ and the involvement of other brain phosphatases. Nonetheless, both PP1 and PP2A inhibition significantly increased pS421-htt and decreased neuronal cell death in vitro. Although these are provocative results, it remains to be seen whether inhibition of these phosphatases can protect neurons in vivo. Finally, a disease-driven decrease in the PP1-inhibitor Darpp-32 and an increase in pS421-htt upon stimulation of the $\mathrm{D}_{1}$ receptor in YAC128 mice suggest a convergence of the dopaminergic and glutamatergic pathways and the phosphorylation state of S421-htt. Together, these experiments demonstrate the neuroprotective role of phosphorylation in HD and encourage further 
investigation of related therapeutic strategies.

\section{References}

Arrasate M, Mitra S, Schweitzer ES, Segal MR, Finkbeiner S (2004) Inclusion body formation reduces levels of mutant huntingtin and the risk of neuronal death. Nature 431:805-810.

Graham RK, Deng Y, Slow EJ, Haigh B, Bissada N, Lu G, Pearson J, Shehadeh J, Bertram L, Murphy Z, Warby SC, Doty CN, Roy S, Wellington CL, Leavitt BR, Raymond LA, Nicholson DW, Hayden MR (2006) Cleavage at the caspase-6 site is required for neuronal dysfunction and degeneration due to mutant huntingtin. Cell 125:1179-1191.

Graham RK, Deng Y, Carroll J, Vaid K, Cowan C, Pouladi MA, Metzler M, Bissada N, Wang L, Faull RL, Gray M, Yang XW, Raymond LA, Hayden MR (2010) Cleavage at the 586 amino acid caspase- 6 site in mutant huntingtin influences caspase- 6 activation in vivo. J Neurosci 30:15019-15029.

Gu X, Greiner ER, Mishra R, Kodali R, Osmand A, Finkbeiner S, Steffan JS, Thompson LM, Wetzel R, Yang XW (2009) Series 13 and 16 are critical determinants of full-length human mutant huntingtin induced disease pathogenesis in HD mice. Neuron 64:828 - 840.

Hardingham GE, Bading H (2010) Synaptic versus extrasynaptic NMDA receptor signaling: implications for neurodegenerative disorders. Nat Rev Neurosci 11:682-696.

Humbert S, Bryson EA, Cordelières FP, Connors NC, Datta SR, Finkbeiner S, Greenberg ME, Saudou F (2002) The IGF-1/Akt pathway is neuroprotective in Huntington's disease and involves Huntingtin phosphorylation by Akt. Dev Cell 2:831-837.

Mansuy IM, Shenolikar S (2006) Protein serine/ threonine phosphatases in neuronal plasticity and disorders of learning and memory. Trends Neurosci 29:679-686.

Metzler M, Gan L, Mazarei G, Graham RK, Liu L, Bissada N, Lu G, Leavitt BR, Hayden MR (2010) Phosphorylation of huntingtin at Ser421 in YAC128 neurons is associated with protection of YAC128 neurons from NMDAmediated excitotoxicity and is modulated by PP1 and PP2A. J Neurosci 30:14318-14329.

Milnerwood AJ, Gladding CM, Pouladi MA, Kaufman AM, Hines RM, Boyd JD, Ko RW, Vasuta OC, Graham RK, Hayden MR, Murphy TH, Raymond LA (2010) Early increase in extrasynaptic NMDA receptor signaling and expression contributes to phenotype onset in Huntington's disease mice. Neuron 65:178-190.

Okamoto S, Pouladi MA, Talantova M, Yao D, Xia P, Ehrnhoefer DE, Zaidi R, Clemente A, Kaul M, Graham RK, Zhang D, Vincent Chen HS, Tong G, Hayden MR, Lipton SA (2009) Balance between synaptic versus extrasynaptic
NMDA receptor activity influences inclusions and neurotoxicity of mutant huntingtin. Nat Med 15:1407-1413.

Pardo R, Colin E, Régulier E, Aebischer P, Déglon N, Humbert S, Saudou F (2006) Inhibition of calcineurin by FK506 protects against polyglutamine-huntingtin toxicity through an increase of huntingtin phosphorylation at S421. J Neurosci 26:1635-1645.

Van Raamsdonk JM, Pearson J, Murphy Z, Hayden MR, Leavitt BR (2006) Wild-type huntingtin ameliorates striatal neuronal atrophy but does not prevent other abnormalities in the YAC128 mouse model of Huntington disease. BMC Neurosci 7:80.

Warby SC, Chan EY, Metzler M, Gan L, Singaraja RR, Crocker SF, Robertson HA, Hayden MR (2005) Huntingtin phosphorylation on serine 421 is significantly reduced in the striatum and by polyglutamine expansion in vivo. Hum Mol Genet 14:1569-1577.

Warby SC, Doty CN, Graham RK, Shively J, Singaraja RR, Hayden MR (2009) Phosphorylation of huntingtin reduces the accumulation of its nuclear fragments. Mol Cell Neurosci 40:121-127.

Zala D, Colin E, Rangone H, Liot G, Humbert S, Saudou F (2008) Phosphorylation of mutant huntingtin at S421 restores anterograde and retrograde transport in neurons. Hum $\mathrm{Mol}$ Genet 17:3837-3846. 